\title{
Incorporation of Potassium at the Pt(111) Surface
}

\author{
J. B. Hannon, M. Giesen, C. Klünker, G. Schulze Icking-Konert, D. Stapel, and H. Ibach \\ Institut für Grenzflächenforschung und Vakuumphysik, Forschungszentrum Jülich, D-52425 Jülich, Germany
}

\section{J.E. Müller}

Institut für Schicht- und Ionentechnik, Forschungszentrum Jülich, D-52425 Jülich, Germany

(Received 8 November 1996)

\begin{abstract}
Using electron-energy-loss spectroscopy and scanning tunneling microscopy, we find that at room temperature the binding site of $\mathrm{K}$ at the $\mathrm{Pt}(111)$ surface changes with increasing coverage. At low coverages, $\mathrm{K}$ adsorbs at surface hollow sites, forming an ionic bond with the substrate. Above a coverage of 0.1 monolayers, we observe a slow migration of $\mathrm{K}$ to subsurface binding sites in the second Pt layer. The measured vibrational frequencies are in quantitative agreement with cluster calculations for the proposed surface and subsurface bonding geometries. [S0031-9007(97)02398-3]

PACS numbers: 71.15.Fv, 71.20.Be, 71.20.Dg, 82.65.My
\end{abstract}

For many years, the interaction of alkali metal (AM) atoms with surfaces was discussed in terms of the chargetransfer model introduced by Gurney [1], which implicitly assumes that the AM atoms adsorb without inducing any morphological changes in the surface. According to this model, the surface bond is due to the partial transfer of the AM $s$ electron to the substrate, which gives rise to a surface dipole and to an AM-AM repulsion that prevents the formation of islands. This picture is the cornerstone of our present understanding of the role of AM as promoters of catalytic reactions, as well as of the mechanism by which the work function is lowered following AM adsorption [2]. However, new results on the adsorption geometry of AM atoms at metal surfaces are beginning to modify this classical picture. Experimental and theoretical investigations have shown that, in the medium- and high-coverage regime, 2D condensation [3] and surface alloy formation [4,5] are favorable under certain circumstances. For low coverages, Lehmann et al. have proposed that $\mathrm{K}$ becomes incorporated into the $\mathrm{Pt}(111)$ surface directly upon adsorption [6]. In this Letter, we also report a subsurface configuration for $\mathrm{K}$ adsorbed at the $\operatorname{Pt}(111)$ surface, but in contrast to the results of Lehmann et al., we show that the subsurface state becomes energetically favorable only for coverages above 0.1 monolayers (ML) [7], and that the conversion to the subsurface site is activated. For coverages below 0.1 ML, we find that $\mathrm{K}$ adsorbs at hollow sites of the surface, with an ionic bond in which one-half of the $\mathrm{K} 4 s$ electron is transferred to the substrate. Our characterization of the K/Pt(111) system was carried out using electronenergy-loss spectroscopy (EELS), scanning tunneling microscopy (STM), Auger electron spectroscopy (AES), and electronic structure calculations using the cluster method.

The experiments were performed using two $\mathrm{Pt}(111)$ crystals, both of which were cut and polished to an accuracy of $0.1^{\circ}$. Well-ordered surfaces were prepared via sputtering with $\mathrm{Ne}^{+}$ions, followed by annealing to
$1250 \mathrm{~K}$. Residual C contamination was removed by exposing the surface to $\mathrm{O}_{2}$ at $950 \mathrm{~K}$, followed by subsequent annealing to $1250 \mathrm{~K}$ to remove the surface oxide layer. $\mathrm{K}$ was evaporated onto the surface with a commercial getter source, and the coverage of the adsorbed $\mathrm{K}$ was determined using AES and low-energy electron diffraction. The evaporation rate of the $\mathrm{K}$ source was determined using the inferred coverage of 0.33 ML for the ordered $\sqrt{3} \times \sqrt{3}$ phase. Our AES calibration is in quantitative agreement with that published by Pirug and Bonzel [8].

We begin by discussing our results for the lowcoverage limit. Figure 1 presents an EEL spectrum recorded after evaporation of $0.02 \mathrm{ML}$ of $\mathrm{K}$ onto the $\mathrm{Pt}(111)$ surface held at $320 \mathrm{~K}$. We assign the intense loss feature at $136 \mathrm{~cm}^{-1}$ to the vibration of $\mathrm{K}$ normal to the surface. Using the coverage and the measured intensity, we derived the cross section for the excitation of the surface vibration [9], which can be conveniently

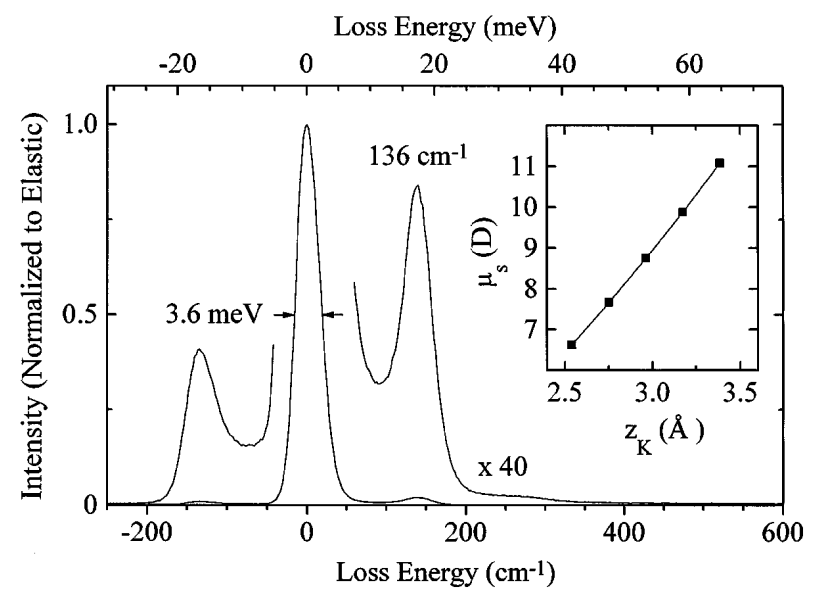

FIG. 1. EEL spectrum recorded after deposition of $0.02 \mathrm{ML}$ of $\mathrm{K}$ on the $\mathrm{Pt}(111)$ surface held at $320 \mathrm{~K}$, showing a single loss at $136 \mathrm{~cm}^{-1}$. The inset shows the calculated surface dipole, $\mu_{s}$, as a function of the adsorption distance, $z_{\mathrm{K}}$, for a $\mathrm{K}$ atom above a hollow site at the $\operatorname{Pt}(111)$ surface. 
expressed in terms of an ionic charge $e^{*}=0.5 \pm 0.2$ electrons per $\mathrm{K}$ atom, with the error arising mainly from the uncertainty in the coverage. The corresponding dynamical dipole moment is given by $d \mu / d z_{\mathrm{K}}=2 e^{*}$, where the factor 2 is due to the effect of the image charge. The spectrum exhibits no discernible changes over a time period of several hours at room temperature, or after heating to $500 \mathrm{~K}$.

In order to aid in the identification of the adsorption site of $\mathrm{K}$ we performed total-energy calculations using the cluster method, which was shown to be well suited to describe localized phenomena at surfaces [10]. The oscillation frequencies and surface dipoles are expected to be converged to about $1 \%$ for the cluster size used in this work. The binding energies require a correct description of the cohesive energy, and may be not quite converged in our clusters, but we expect their relative values at different sites of the same cluster (or of a cluster of similar size and shape) to be of useful accuracy. We use the Kohn-Sham scheme with the local density approximation (LDA) for exchange and correlation [11], which has been shown to provide a correct description of the interaction between AM atoms and metal surfaces $[5,12,13]$. The one-electron problem was solved using a localized muffin-tin orbital basis [14], including $s, p$, and $d$ functions for all atoms. Spin effects were neglected and the core electrons kept frozen, but the Pt $5 d$ electrons, as well as the $\mathrm{K} 4 s$ and $3 p$ electrons, were incorporated in the calculation. We used a $\mathrm{Pt}_{25} \mathrm{~K}$ cluster consisting of two Pt layers: a $\mathrm{Pt}_{12}$ layer and a $\mathrm{Pt}_{13}$ layer, having at their center a threefold hollow site and an on-top site, respectively [Fig. 2(a)]. Adsorption at the hollow site was studied by placing the $\mathrm{K}$ atom above the center of the $\mathrm{Pt}_{12}$ surface and calculating the total energy of the cluster as a function of the height of the $\mathrm{K}$ atom, $z_{\mathrm{K}}$. The potential energy was obtained by subtracting the energy of the $\mathrm{Pt}_{25}$ cluster. The minimum of the potential energy function yields an equilibrium height of $2.8 \AA$ above the surface and an adsorption energy, $E_{s}=-2.45 \mathrm{eV}$, in good agreement with thermal desorption measurements of Garfunkel and Somorjai [15], who derived an adsorption

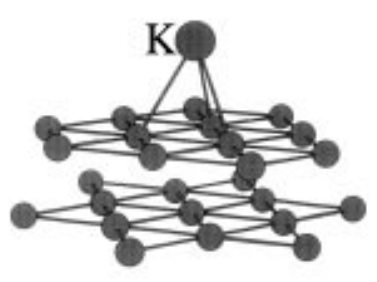

(a)

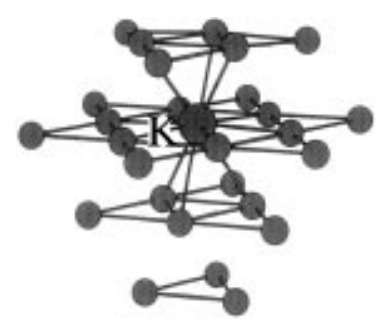

(b)
FIG. 2. (a) $\mathrm{Pt}_{25} \mathrm{~K}$ cluster utilized in the calculations to model a $\mathrm{K}$ atom adsorbed at the hollow site of the $\mathrm{Pt}(111)$ surface. (b) $\mathrm{Pt}_{27} \mathrm{~K}$ cluster employed to model a $\mathrm{K}$ atom embedded in the second Pt layer of the same surface. energy of $2.6 \mathrm{eV}$ per $\mathrm{K}$ atom for the zero-coverage limit. Adsorption at the on-top site, which was studied by placing the $\mathrm{K}$ atom at the $\mathrm{Pt}_{13}$ side of the cluster, was found to be unfavorable by $140 \mathrm{meV}$ compared with the hollow site. We also considered surface alloy geometries in which $\mathrm{K}$ is bound either in a single Pt-atom vacancy, or in the center of a three-Pt-atom vacancy island. These geometries are unfavorable compared to the flat-surface hollow site by 0.6 and $0.9 \mathrm{eV}$, respectively. These results lead us to exclude random surface alloy formation in the low-coverage regime.

From the calculated potential energy functions we deduce oscillation frequencies of $138 \mathrm{~cm}^{-1}$ for the hollow site (in excellent agreement with our experimental value of $\left.136 \mathrm{~cm}^{-1}\right), 149 \mathrm{~cm}^{-1}$ for the on-top site, $100 \mathrm{~cm}^{-1}$ for the single-atom vacancy, and $125 \mathrm{~cm}^{-1}$ for the threeatom vacancy. These results confirm the assignment of the hollow site as the only occupied site at low coverages.

The inset to Fig. 1 shows the calculated surface dipole, $\mu_{s}=\mu\left(\mathrm{Pt}_{25} \mathrm{~K}\right)-\mu\left(\mathrm{Pt}_{25}\right)$, as a function of $z_{\mathrm{K}}$ for the hollow site. At equilibrium we find $\mu_{s}=7.8$ debye (in good agreement with the initial dipole deduced from work function measurements [16]). The dynamical dipole of the vibration is given by $d \mu_{s} / d z_{\mathrm{K}}=1.1$ electrons (to be compared with the value of 1.0 electrons derived from the measured EELS intensity). Note that to a very good approximation $\mu_{s}=2 e^{*} z_{\mathrm{K}}+$ const, i.e., $\mu_{s}$ varies almost linearly with the height of the $\mathrm{K}$ atom above the surface. This means that when the adsorption height is changed, the surface dipole behaves as if there was a fixed charge, $e^{*}$, attached to the $\mathrm{K}$ atom: The bond is ionic.

With increasing coverage the vibration frequency of adsorbed $\mathrm{K}$ increases linearly to a maximum value of $180 \mathrm{~cm}^{-1}$ at $0.15 \mathrm{ML}$ (presumably due to dipole-dipole interactions), while its dynamical dipole moment decreases in the same coverage range to 0.3 electrons. For coverages above $0.15 \mathrm{ML}$, the dynamical dipole remains constant, but the frequency of the loss decreases with increasing coverage, indicating a change in the electronic structure of the overlayer. For coverages of $\mathrm{K}$ above $0.1 \mathrm{ML}$ we observe an additional loss feature in EELS, which grows in intensity as a function of time. Figure 3 shows a series of EEL spectra recorded at approximately 30 minute intervals following the deposition of $0.13 \mathrm{ML}$ of $\mathrm{K}$ onto the surface held at $320 \mathrm{~K}$. Immediately after evaporation, we observe a single loss feature at $180 \mathrm{~cm}^{-1}$, due to the vibration of $\mathrm{K}$ adsorbed in hollow sites at the surface, shifted upwards from $136 \mathrm{~cm}^{-1}$ by dipole-dipole interactions. After about 40 minutes, an additional feature appears at $225 \mathrm{~cm}^{-1}$, which gradually develops into the dominant feature of the spectrum. The fact that the new feature grows at the expense of the $180 \mathrm{~cm}^{-1}$ loss, and that the energy of the new loss lies above the top of the Pt phonon band $\left(\omega_{\max } \sim 200 \mathrm{~cm}^{-1}\right)$, strongly suggests that the $225 \mathrm{~cm}^{-1}$ loss must be associated with $\mathrm{K}$ in a new binding site. 


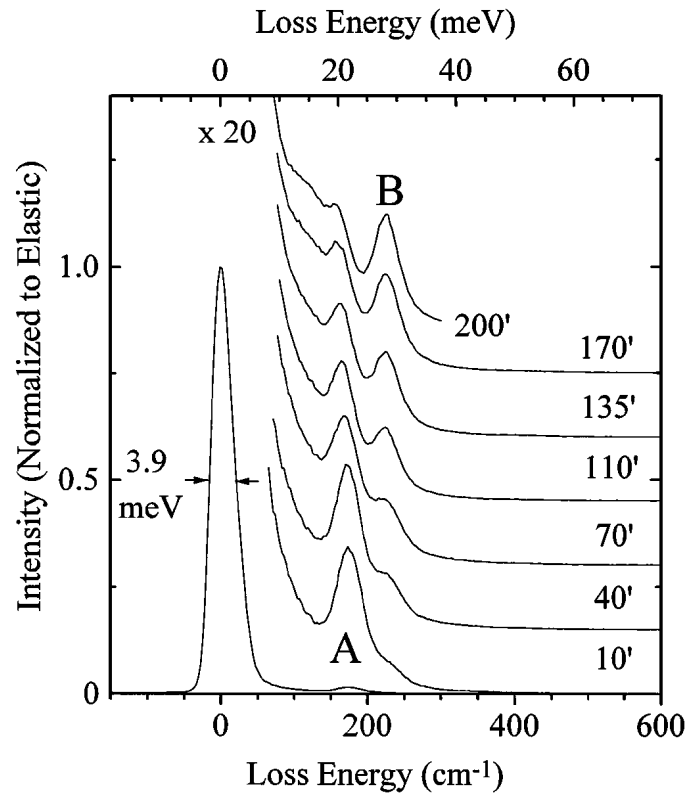

FIG. 3. Time development of the EEL spectrum recorded following the deposition of $0.13 \mathrm{ML}$ of $\mathrm{K}$ on the $\mathrm{Pt}(111)$ surface held at $320 \mathrm{~K}$. We identify the losses at $180 \mathrm{~cm}^{-1}$ (A) and $225 \mathrm{~cm}^{-1}$ (B) as adsorbed and embedded $\mathrm{K}$, respectively.

Before we can identify the $225 \mathrm{~cm}^{-1}$ feature as $\mathrm{K}$ bound in a second site, we must exclude the possibility of residual contamination. Fortunately, there are several EELS studies of the coadsorption of simple molecules with $\mathrm{K}$ at the $\mathrm{Pt}(111)$ surface. Comparison with these studies allows us to exclude $\mathrm{CO}$ [17], $\mathrm{CO}_{2}$ [18], and $\mathrm{H}_{2} \mathrm{O}$ [19] as possible sources of contamination. We investigated the possibility of coadsorption with $\mathrm{O}$ by exposing the $\mathrm{K} / \mathrm{Pt}(111)$ surface $(0.15 \mathrm{ML}$ of $\mathrm{K})$ to 1.2 Langmuir of $\mathrm{O}_{2}$ at room temperature. Three $\mathrm{O}$-induced losses appear in the range $450-600 \mathrm{~cm}^{-1}$, however, no change in the loss at $225 \mathrm{~cm}^{-1}$ loss is observed. Carbon contamination, induced by prolonged exposure of the surface to a hot $\mathrm{W}$ filament, gives rise to losses in the range $550-650 \mathrm{~cm}^{-1}$, in agreement with total-energy cluster calculations for coadsorbed $\mathrm{K}$ and $\mathrm{C}$ at the $\mathrm{Pt}(111)$ surface [20].

The frequency of the $225 \mathrm{~cm}^{-1}$ loss is nearly a factor of $\sqrt{2}$ greater than that of a single $\mathrm{K}$ atom bound at a surface site. This implies that the number of vertically oriented K-Pt bonds in the new geometry is roughly double that of surface-bonded $\mathrm{K}$, suggesting that the new loss is associated with a $\mathrm{K}$ atom bound in a subsurface vacancy. Furthermore, the fact that the new state is observable in EELS indicates that the $\mathrm{K}$ must be localized near the surface. Appreciable diffusion of $\mathrm{K}$ into the bulk is also ruled out by AES measurements, which show no discernible change in $\mathrm{K}$ coverage over the time scale of several hours (at $300 \mathrm{~K}$ ), or upon heating of the surface to $550 \mathrm{~K}$. Without speculating on the reaction path into the subsurface site, it is clear that the conversion to the new state must involve an activated process because of the large delay in the appearance of the new feature after deposition of $\mathrm{K}$.

From Fig. 3 it is apparent that a large fraction of the $\mathrm{K}$ monolayer converts to the subsurface geometry. This can also be seen in Fig. 4, which shows a differentiated STM image from the atomically resolved $\operatorname{Pt}(111)$ surface with $0.12 \mathrm{ML}$ of $\mathrm{K}$ adsorbed at $300 \mathrm{~K}$. Although the periodicity of the $\mathrm{Pt}(111)$ lattice is preserved, the lattice is distorted vertically in localized regions (visible as brighter areas in the image). We attribute these features to a local outward buckling of the surface layer due to the presence of subsurface $\mathrm{K}$. Because the diffusion barrier of $\mathrm{K}$ at the $\mathrm{Pt}(111)$ surface is only about $25 \mathrm{meV}$ [21], surface $\mathrm{K}$ atoms diffuse too rapidly to be observed in STM images at room temperature. In addition to the buckled areas, STM images show a significant restructuring of the steps upon adsorption of $\mathrm{K}$. The straight steps of the clean surface become jagged, perhaps suggesting that the incorporation of $\mathrm{K}$ takes place at steps.

If the $\mathrm{Pt}(111) / \mathrm{K}$ system is heated to $500 \mathrm{~K}$, the $225 \mathrm{~cm}^{-1}$ loss disappears from the EEL spectrum, and the intensity of the lower frequency mode reverts to the initial value, indicating that the surface goes back to the configuration it had immediately after evaporation. When the sample is recooled to $320 \mathrm{~K}$, the $225 \mathrm{~cm}^{-1}$ loss again gradually replaces the lower frequency mode, and the time development repeats itself as illustrated in Fig. 3. This shows that, while the subsurface state has lower energy, the adsorbed state has larger entropy which dominates the free energy for sufficiently high temperature. The large entropy arises from the extremely low frequency of the parallel vibration of adsorbed $\mathrm{K}, \omega_{\|} \sim 50 \mathrm{~cm}^{-1}$ [21]. From this frequency we estimate the difference in the free energy between 300 and $500 \mathrm{~K}$ to be about $0.1 \mathrm{eV}$ [22].

The picture of a $\mathrm{K}$ atom bound in a subsurface vacancy is confirmed by total-energy cluster calculations. We modeled the incorporation process by exchanging a $\mathrm{Pt}$ atom in the interior of an appropriate cluster with a $\mathrm{K}$

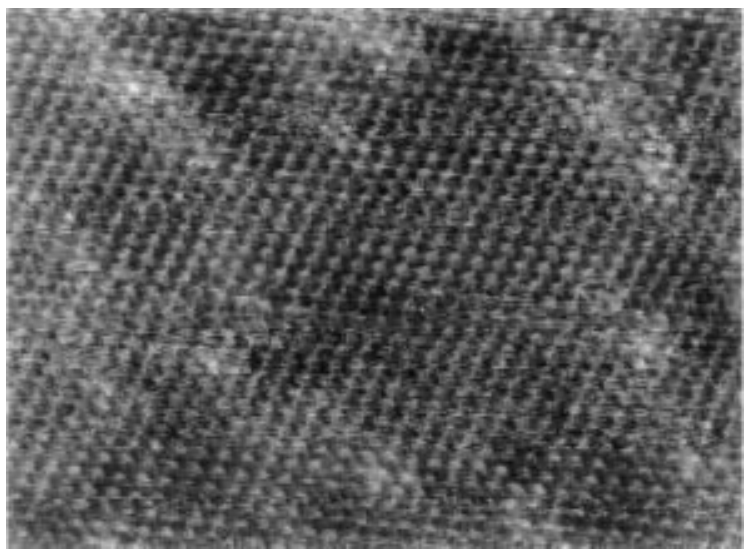

FIG. 4. Differentiated STM image of the Pt(111) surface recorded after deposition of $0.12 \mathrm{ML}$ of $\mathrm{K}$ at $300 \mathrm{~K}$. The dimensions of the imaged region are $120 \AA \times 90 \AA$. 
atom brought from infinity, and placing the Pt atom at a kink site at the surface of the cluster. We employed the $\mathrm{Pt}_{27} \mathrm{~K}$ cluster shown in Fig. 2(b), consisting of a $\mathrm{Pt}_{12} \mathrm{~K}$ layer with the $\mathrm{K}$ atom at the center, sandwiched between a $\mathrm{Pt}_{6}$ layer above and two layers below, a $\mathrm{Pt}_{6}$ layer and a $\mathrm{Pt}_{3}$ layer. After a restricted relaxation of the cluster, we find that the $\mathrm{K}$ atom binds $0.05 \AA$ above the plane of the second layer and that the three Pt atoms above the $\mathrm{K}$ atom move upwards by $0.2 \AA$, which is consistent with the outward buckling observed with STM. For this geometry we calculated the vibrational frequency of the $\mathrm{K}$ atom to be $230 \mathrm{~cm}^{-1}$, in excellent agreement with the experimental value. This oscillation frequency was also obtained using smaller clusters with the same coordination for the $\mathrm{K}$ atom.

The calculations predict that subsurface $\mathrm{K}$ does not affect the work function of the surface (i.e., $\mu_{b}=0$ ), and that its dynamical dipole is given by $d \mu_{b} / d z_{\mathrm{K}}=0.1$ electrons. This is about half of the value required to account for the intensity of the $225 \mathrm{~cm}^{-1}$ loss in Fig. 3 (for comparison, the initial dynamical dipole of the low frequency loss is 0.3 electrons). Although we cannot exclude a numerical error due to cluster-size effects, measurements at higher impact energies indicate that the large intensity of the $225 \mathrm{~cm}^{-1}$ loss is due to an enhanced contribution of impact scattering. We observed that when the impact energy is increased from 5 to $15 \mathrm{eV}$, the intensity of the low frequency mode decreases more than that of the $225 \mathrm{~cm}^{-1}$ mode, which means that the latter has a larger contribution from impact scattering.

The condition for incorporation of $\mathrm{K}$ is given by

$$
E_{b}<E_{s},
$$

where $E_{s}$ is the adsorption energy at the surface and $E_{b}$ the embedding energy in the subsurface state. We calculate $E_{b}=E\left(\mathrm{Pt}_{27} \mathrm{~K}\right)-E\left(\mathrm{Pt}_{26} \mathrm{Pt}\right)-E(\mathrm{~K})=-1.3 \mathrm{eV}$, where the reference $\mathrm{Pt}_{26} \mathrm{Pt}$ cluster was constructed starting from the $\mathrm{Pt}_{27} \mathrm{~K}$ cluster, and replacing the $\mathrm{K}$ atom by a Pt atom taken from a kink site. In the low-coverage limit, $E_{s}=-2.45 \mathrm{eV}$, and incorporation is not favorable. However, with increasing coverage, the repulsive K-K interaction leads to a decrease of the adsorption energy, such that above a critical coverage condition (1) becomes satisfied, and part of the overlayer is transferred from the surface hollow site to the subsurface geometry. The embedding energy, $E_{b}$, is the result of a trade-off between the energy gain due to the increased coordination of the $\mathrm{K}$ atom in the subsurface geometry, and the energy penalty associated with producing the vacancy and the distortion of the Pt lattice surrounding the embedded K. We specu- late that the energy cost of distorting the lattice is larger in the bulk than near the surface, where the coordination of the Pt atoms is reduced, and we believe that this is the effect that stabilizes the embedded $\mathrm{K}$ state near the surface. We note, however, that there is experimental evidence from STM images that some K goes into the bulk at high temperatures when the surface has been exposed to higher coverages.

We are indebted to $\mathrm{U}$. Linke for the preparation of the Pt samples, and S. Lehwald and G. Pirug for useful discussions.

[1] R. W. Gurney, Phys. Rev. 47, 479 (1935).

[2] For a review, see Physics and Chemistry of Alkali Metal Adsorption, edited by H. P. Bonzel, A. M. Bradshaw, and G. Ertl (Elsevier, Amsterdam, 1989).

[3] S. Modesti, C. T. Chen, Y. Ma, G. Meigs, P. Rudolf, and F. Sette, Phys. Rev. B 42, 5381 (1990).

[4] C. Stampfl, M. Scheffler, H. Over, J. Burchhardt, M. Nielsen, D. L. Adams, and W. Moritz, Phys. Rev. Lett. 69, 1532 (1992).

[5] J. Neugebauer and M. Scheffler, Phys. Rev. Lett. 71, 577 (1993).

[6] J. Lehmann, P. Roos, and E. Bertel, Phys. Rev. B 54, R2347 (1996).

[7] We define the coverage as the number of $K$ atoms divided by the number of surface Pt atoms: $1 \mathrm{ML}=$ 0.15 atoms $/ \AA^{2}$.

[8] G. Pirug and H. P. Bonzel, Surf. Sci.. 194, 159 (1988).

[9] H. Ibach and D.L. Mills, Electron Energy Loss Spectroscopy and Surface Vibrations (Academic Press, New York, 1982).

[10] J. E. Müller, Surf. Sci. 178, 589 (1986).

[11] W. Kohn and L. J. Sham, Phys. Rev. 140, A1133 (1965).

[12] N. D. Lang, Phys. Rev. B 4, 4239 (1971).

[13] E. Wimmer, A. J. Freeman, J. R. Hiskes, and H. M. Karo, Phys. Rev. B 28, 3074 (1983).

[14] J. E. Müller, R. O. Jones, and J. Harris, J. Chem. Phys. 79, 1874 (1983).

[15] E. L. Garfunkel and G. A. Somorjai, Surf. Sci. 115, 441 (1982).

[16] M. Kisikinova, G. Pirug, and H. P. Bonzel, Surf. Sci. 133, 321 (1983).

[17] G. Pirug and H. P. Bonzel, Surf. Sci. 199, 371 (1988).

[18] J. M. White, in Ref. [2].

[19] P. Baumann, G. Pirug, D. Reuter, and H. P. Bonzel, Surf. Sci. 335, 186 (1995).

[20] J. E. Müller (unpublished).

[21] J.E. Müller in The Chemical Physics of Solid Surfaces, edited by D. A. King and D.P. Woodruff (Elsevier, Amsterdam, 1993), Vol. 6.

[22] A. Grossmann, W. Erley, and H. Ibach, Phys. Rev. Lett. 71, 2078 (1993). 